\title{
Segmentation of Phonocardiograms Signal
}

\author{
Jamuna Kaushik $^{1}$ and Abhishek Misal ${ }^{2}$ \\ M. Tech Scholar ${ }^{1}$ and Sr. Assistant Professor ${ }^{2}$ \\ Department of Electronics \& Telecommunication \\ Chhatrapati Shivaji Institute of Technology \\ Durg, Chhatisgarh \\ India
}

\begin{abstract}
Heart sound is a kind of bio-sound, mainly through the media pass sound signals. The measures of the heart sound signals involved in acoustics, fluid mechanics research. when added some noise for selecting pure PCG signal by using adaptive white Gaussian noise. Than denoise the PCG signal by using Discrete Wavelet transform and decomposes a signal into a 4 level of basic functions. These basic functions are called Discrete wavelet transform (DWT), which transforms a discrete time signal to a discrete wavelet representation. Experiments are conducted on 23 different recordings of heart sound where experiment working on 2 normal signal and 19 Abnormal signals where working on the accuracy of the PCG signal that is depending on the training and testing data. Segmentation process based on Shannon entropy method for low amplitude and GSF is based on high amplitude. Toward this objective, after preprocessing the PCG signal, for feature extraction of the PCG signal fixed windows were moved on the preprocessed signal, and in each analysis window, two frequency-and amplitude-based features were calculated from the excerpted segment. In order to recognize the delineated PCG sounds, first, S1 and S2 were detected. Then, a new DS was regenerated from the signal whose $S 1$ and $S 2$ were eliminated to detect occasional $S 3$ and $S 4$ sounds. Finally, probable murmurs and souffles were spotted. The proposed algorithm was applied to 6 beat PCG. signals gathered from patients with different valve diseases. This feature Extraction of the PCG signal is use PCA for reducing 216 dimensions to 7 dimensions of the PCG signal. and the classifier is used SVM method which is found the normal and abnormal heart sound of the PCG signal.
\end{abstract}

Key Words: PCG, DWT, Segmentation, classification, PCA, SVM.

\section{INTRODUCTION}

Cardiovascular diseases are the world's largest killers taking 17.1 million lives a year. Although, there have been a lot of developments in the land of cardiac technologies, the practices of stethoscope and cardiac auscultation persist ancient diagnostic tool in cardiac pathology calculation and the connected mechanical functions. Heart sound auscultation is a helpful and Noninvasive diagnostic tool for detecting heart valves' abnormalities. Also, heart sound analysis has been used to spot heart diseases. A major problem in heart sound analysis is to robustly detect the location of the honest sound (called detection) and its onsetoff set spots (called delineation or segmentation) during each cardiac cycle in the presence of severe heart valvular disorders, heart diseases, highlevel measurement noises, and circumferential disturbances.

\section{METHODOLOGY}

The methods presented here are divided into fourth modules. First the heart sound is pre-processed and the possible candidates for S1 or S2 are selected. Secondly, features for each of the selected candidates are extracted. Third PCA is dimension reduce for classification and feature extraction. Finally, the candidates are classified as one of the possible outcomes. 


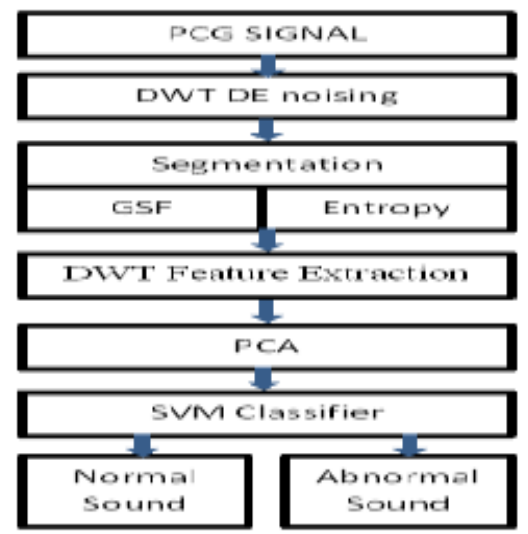

Figure 1. Proposed methodology

\subsection{FILTERING ALGORITHM}

The PCG signal, acquired through phonocardio-transducer usually contains a lot of noise. The hardware gaining system of PCG signal has been equipped with de-noising function at present, but it can't dispose the noise completely. Therefore, heaviness is placed on applying the digital signal processing technology to PCG signal de-nosing. Owing to the difference for each method's strength and weakness, discrete wavelet transform de-nosing approach has been used generally. This paper describes an improved DWT de-noising method for the PCG signal processing.

\subsection{DISCRETE WAVELET TRANSFORM}

Wavelets show greater signal denoising concert due to their properties such as multi resolution and windowing. This project focuses on denoising of phonocardiogram (PCG) signals using different families of discrete wavelet transforms, using soft thresholding types and techniques, and signal decomposition fourth levels. In particular, we discuss the effect of the preferred wavelet function and wavelet decomposition level on the efficiency of the denoising algorithm. Denoised signals are compared with the original PCG signal to determine the most suitable parameters (wavelet family, level of decomposition, and thresholding type) for the denoising process .In this paper heursure method is best denoise method at the level of fourth.

\subsection{SEGMENTATION}

In the project segmentation of the signal is high and low amplitude for high amplitude envelope decide used GSF and low amplitude use envelope decide Shannon entropy method.

\subsection{GAUSSIAN SMOOTHING FILTER (GSF)}

In signal giving out applications, when an analysis window is applied for a positive purpose, the length of the window should be careful as a critical and sensitive design parameter. In other words, several indices such as sampling frequency, acquisition bitrate, signal-to-noise ratio, extent of frequency component, external disturbances, and some other influencing factors have direct eff ects on the eff ectiveness of the affecting window. This sensitivity to a lot of parameters often imposes a drawback to algorithms which use analysis windows. The Gaussian weighting technique described in this study, is aimed to decrease the dependency of the analysis performance (envelope exteraction) on the length of the window In order to cast the GSF problem into a mathematical formulation, a sliding window with length $1 \mathrm{~ms}$ is selected, and the value of mid-sample of the window is replaced by the average value of Gaussian-weighted signal.smoothing filter using noise redusing filter of the time and frequency domain by the fix window length. When rms value is less than mean value Gaussion smoothing filter is work for selecting high amplitude cofficients signal. GSF is given equation:

$$
\begin{aligned}
\operatorname{GSF}[\mathrm{s}(\mathrm{t}), 1, \sigma, \mathrm{k}] & =\mathrm{GSF}[\mathrm{GSF}[\mathrm{s}(\mathrm{t}), 1, \sigma, \mathrm{k}-1], 1, \sigma, 1] \\
\mathrm{GSF}[\mathrm{s}(\mathrm{t}), 1, \sigma, 1] & =\int_{t-\frac{l}{2}}^{t+\frac{l}{2}} s(\tau) \times \frac{1}{\sqrt{2 \pi \sigma^{2}}} \exp \left(-\frac{(\tau-t)^{2}}{2 \sigma^{2}}\right) d \tau---(1)
\end{aligned}
$$




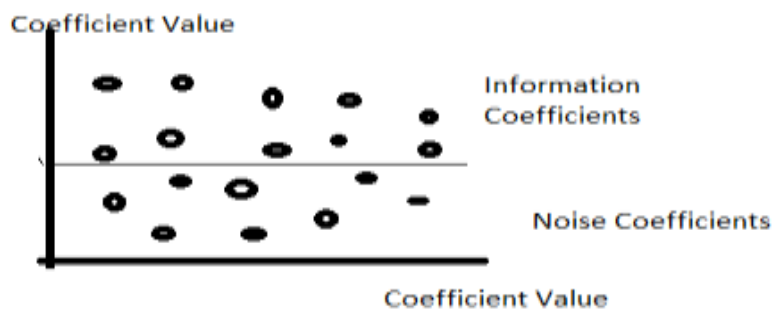

Figure 2. Gaussion smooth filter

\subsection{Shannon Entropy}

After preprocessing, we extract the Shannon envelope of the normalized signal. The average Shannon energy is calculated fixed window length. A predefined function was then used to detect peaks of S1 and S2 heart sounds while founding on an amplitude threshold. Thereafter, some extra peaks are rejected when the distance to the nearest peak is smaller than a distance threshold. Other extra peaks are rejected and the distinction between S1 and S2 is performed based on the fact that diastolic period is greater than systolic period if parameter value of rms is greater than mean value than low amplitude entropy condition is used.

Square of the energy:

$$
\mathrm{E}=S^{2}(\mathrm{t})
$$

Absolute value of the energy:

$$
\mathrm{E}=|S(t)|
$$

Shannon entropy:

$$
\mathrm{E}=-|\mathrm{S}(\mathrm{t})|^{*} \log |\mathrm{S}(\mathrm{t})|
$$

Shannon energy:

$$
E=-S 2(t) * \log S 2(t)
$$

\subsection{Principal Component Analysis (PCA)}

In this paper, we proposed a robust segmentation algorithm using principal component analysis (PCA) which reduces the computational simplicity.Principal component analysis (PCA) is a method for analysing data sets of high dimension, tightfitting patterns and highlighting the similarities and differences. Although PCA can be used for different types of investigation, here the emphasis is on data reduction and feature extraction. The purpose of using this is to remove dismissed information and replace a group of variables which measures the same information with a single new variable called principal component (PC). The calculation of the PC is principally equivalent to performing the singular value decomposition (SVD) on a data set.

\subsection{Properties of PCA:}

There are lot of number of ways to determine an orthogonal basis for a particular set of data. Based on its properties PCA provides useful benefits in feature extraction problems.

The properties of PCA are:

1) Each PC contains line combination of original variables.

2) All the PCs are orthogonal to each other so there is no redundant information.

3) The PC's as a entire form an orthogonal basis for the space of the data. Each principal Eigen value measures the amount of information captured in the direction of a corresponding Eigen vector. In this sense, the original data set is transformed so that it is expressed in terms of patterns between the variables. 
Jamuna Kaushik et. al., Segmentation of Phonocardiograms Signal

\subsection{Classifier}

In this project SVM is classified normal and abnormal heart sound signal from the 7 dimension data.

\section{SVM(Support Vector Machine):}

1.Support Vector Machine (SVM) is a discriminative classifier formally defined by a separating hyperplane. In two dimentional space this hyperplane is a line dividing a plane in two parts where in each class lay in either side.

2.Suppose you are given plot of two label classes on graph as shown in fig

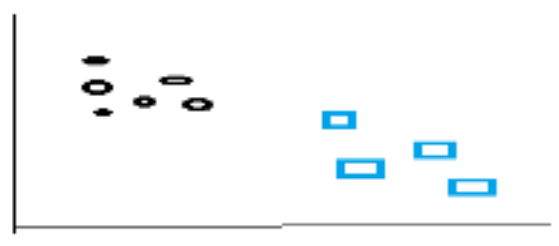

\section{Image 1-Draw a line that separates black circles and blue squares.}

3.It fairly separates the two classes. Any point that is left of line falls into black circle class and on right falls into blue square class. Separation of classes.

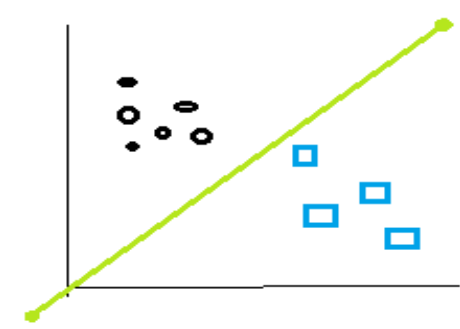

\section{Image 2- Sample cut to divide into two classes.}

4. We apply transformation and add one more dimension as we call it $\mathrm{z}$-axis. Lets assume value of points on $\mathrm{z}$ plane, $\mathrm{w}=\mathrm{x}^{2}+$ $\mathrm{y}^{2}$.

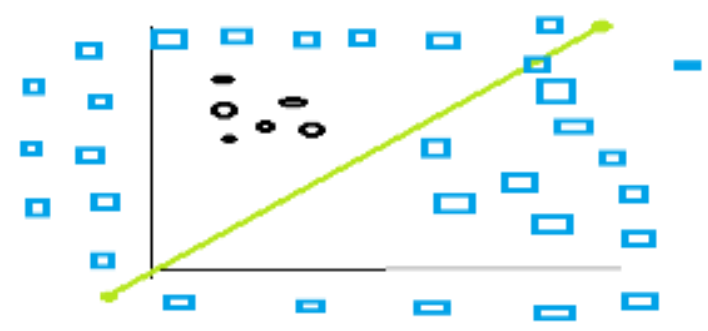

\section{Image 3. Draw a separating line}

5. When we transform back this line to original plane, it maps to circular boundary as shown in image 4. These transformations are called kernels. 


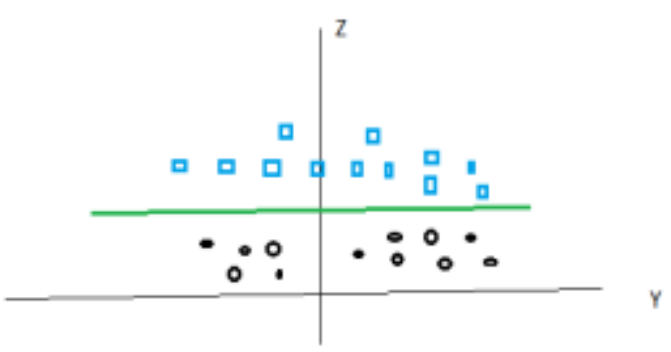

Figure 4. Selected original plane

\section{RESULT AND DISCUSSION}

There is a need for a fast and reliable method to detect the presence of noise in PCG signals that will allow an accurate interpretation of heart sounds and diagnosis of cardiac disorders. Various authors presented a novel low-complex and multi-channel methodology for the detection of noise, which is based on the time and frequency domain analysis of the PCG signal. The multichannel approach is able to achieve high performance, with low computational complexity. The method is important for the comparison of the proposed algorithm with other noise detection algorithms used to analyze PCG signals and finally on the evaluation of the algorithm in a larger population consisting of both healthy and cardiovascular disease subjects.

\subsection{Output Of Heasure Method :}

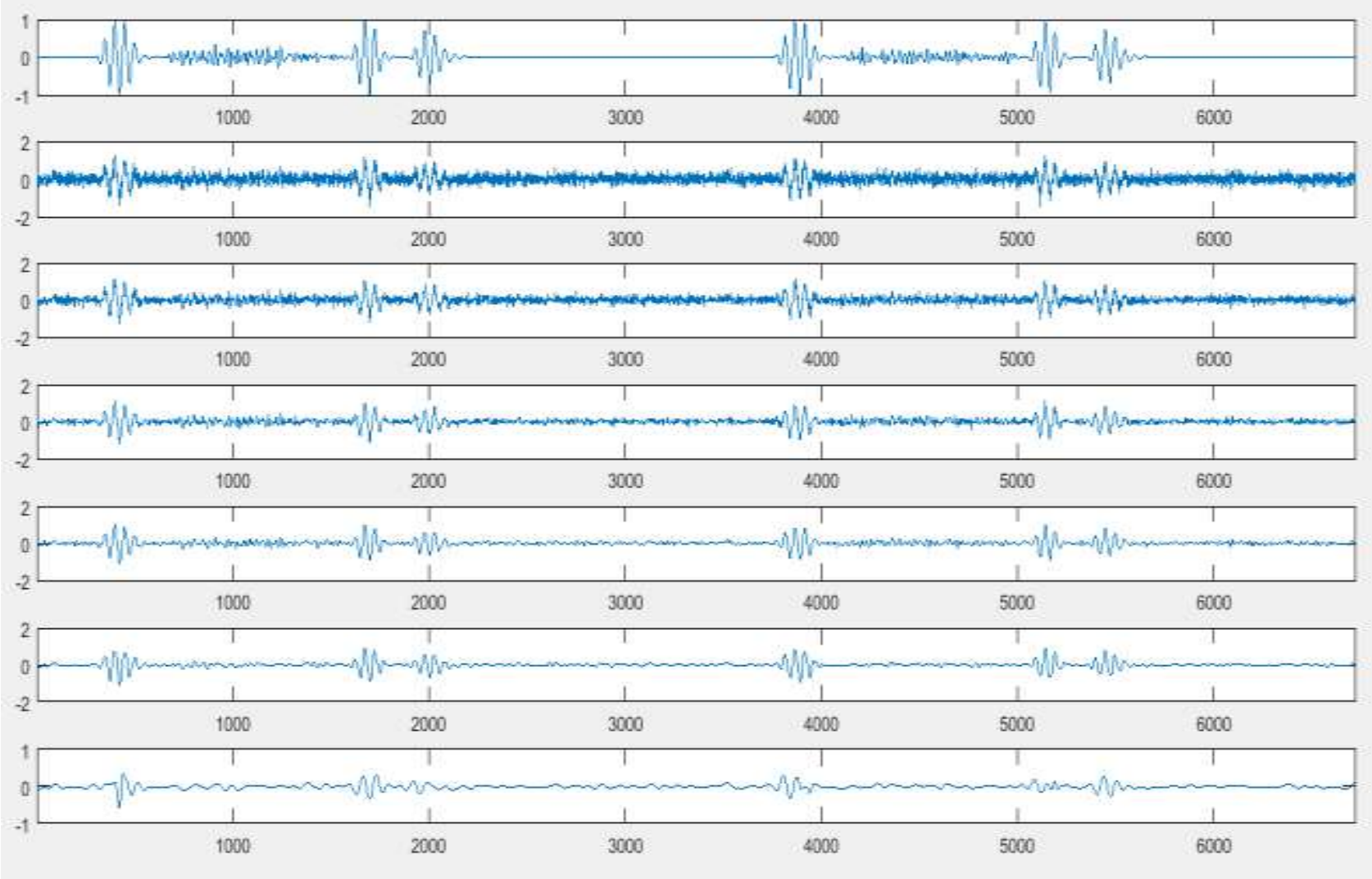

Fig.3.1 -(a) Original pcg signal (b) noisy pcg signal (c)denoise pcg signal (d)level 1 (e)level 2(f) level 3(g)level 4 
Jamuna Kaushik et. al., Segmentation of Phonocardiograms Signal

\subsection{Output Of Minimaxi Method :}

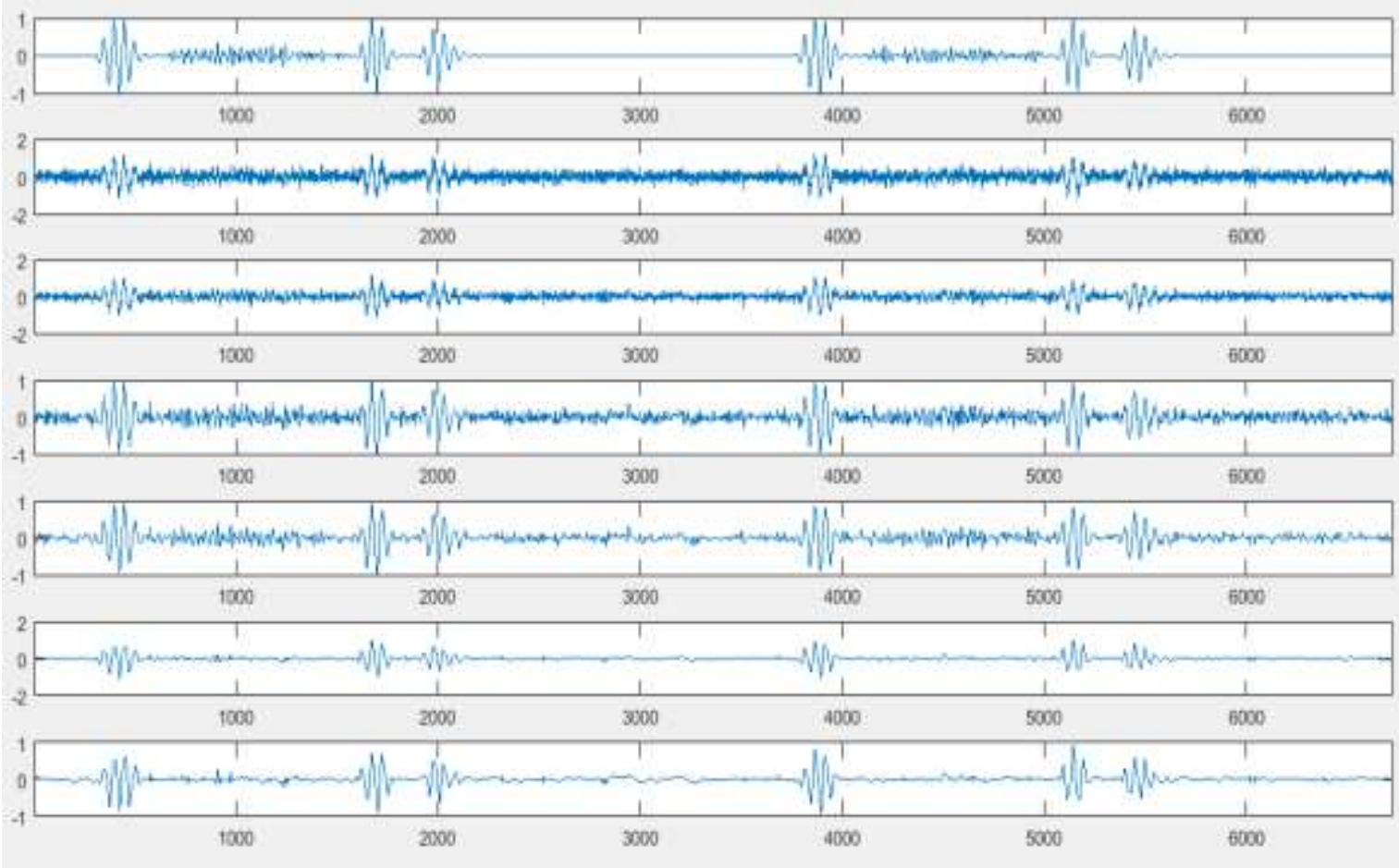

Fig.3.2 -(a) Original pcg signal (b) noisy pcg signal (c)denoise pcg signal (d)level 1 (e)level 2(f) level 3(g)level 4

\subsection{Output Of Square Method}

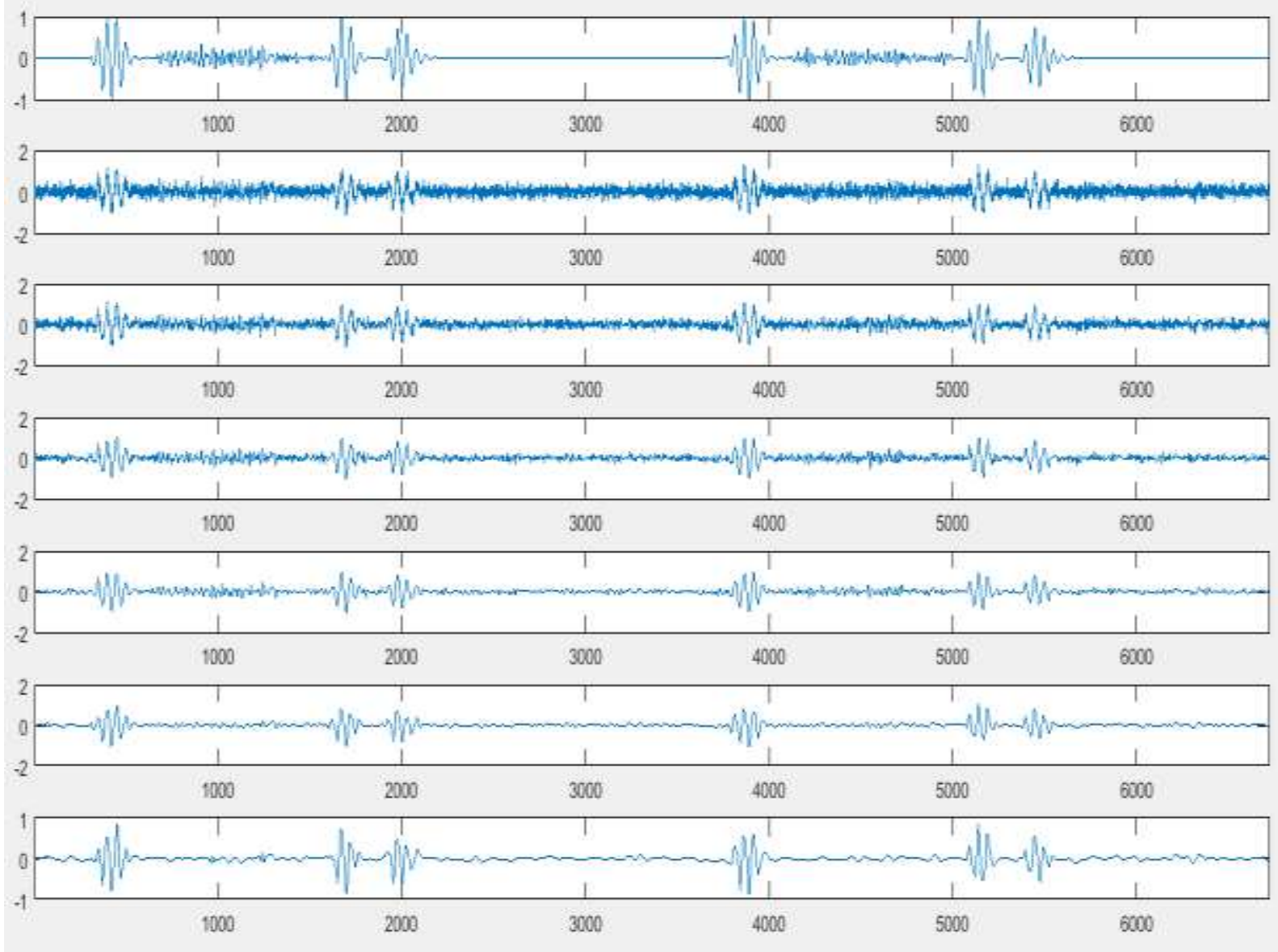

Fig.3.3 -(a) Original pcg signal (b) noisy pcg signal (c)denoise pcg signal (d)level 1 (e)level 2(f) level 3(g)level 4 
International Journal of Engineering Research And Advanced Technology, Vol.4, Issue 7, July-2018

heasure soft thesolding method is a best denoise method at the level 4 when add $1 \mathrm{db}$ noise, SNR value is 0.99 .

\subsection{GSF Based Detected S1 And S2 Heart Sound}
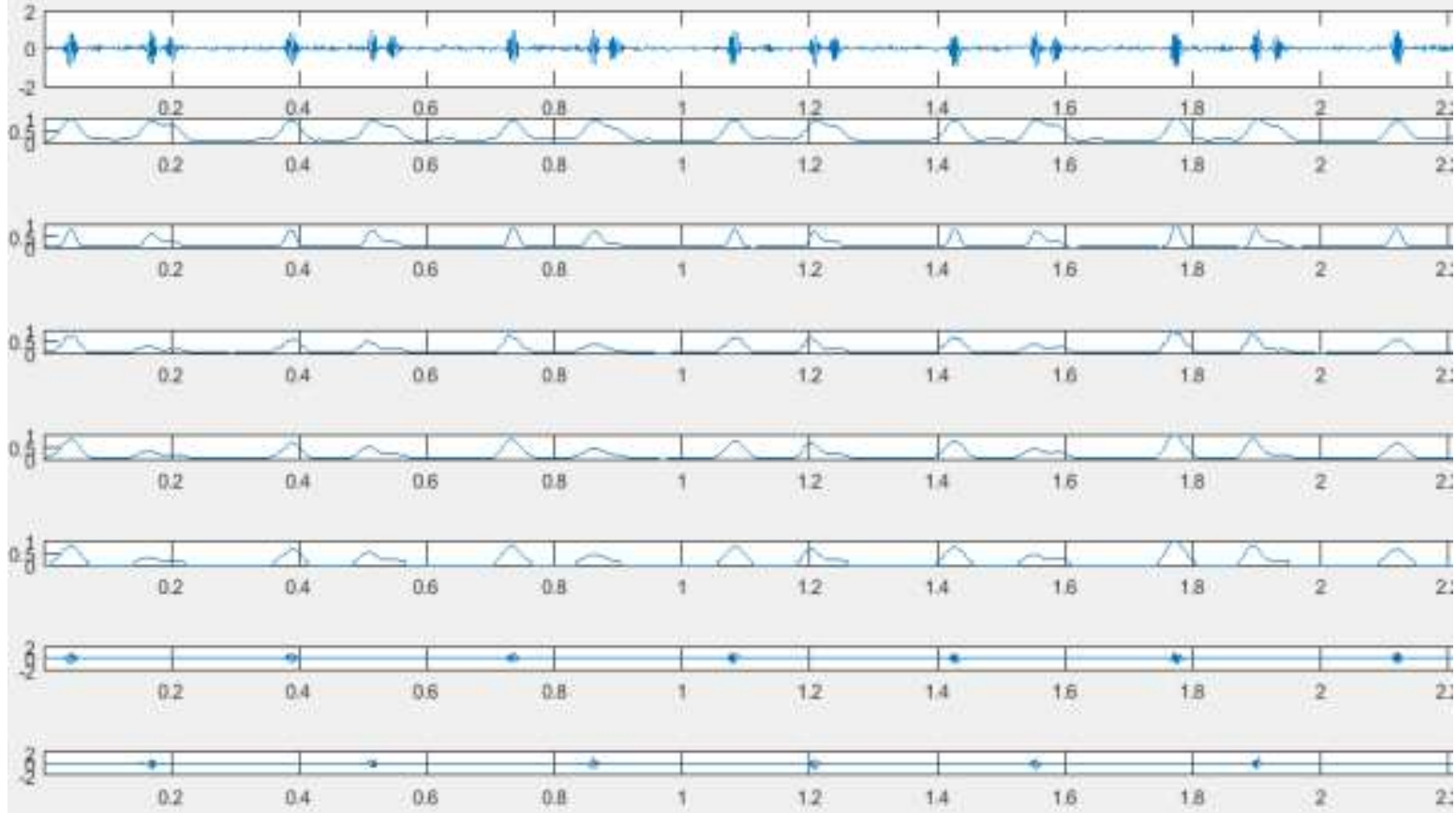

Fig.3.4 - (a) Denoise pcg signal (b)Time domain envelope (c)Frequency domain envelope(d) multiply time and frequency domain (f) apply GSF filter(g)detected s1(h)detected s2

\subsection{Applying Threshold Level}

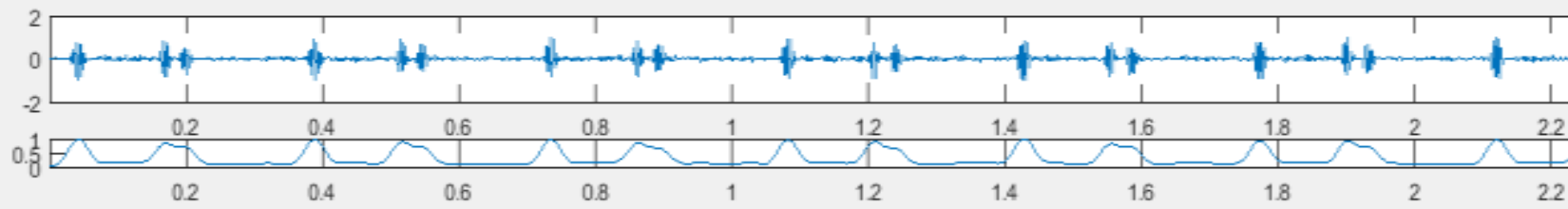

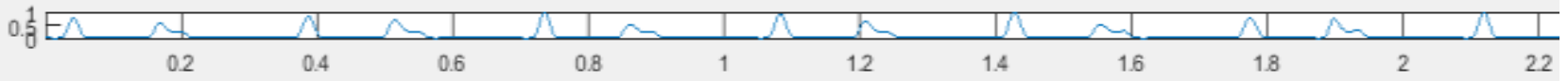

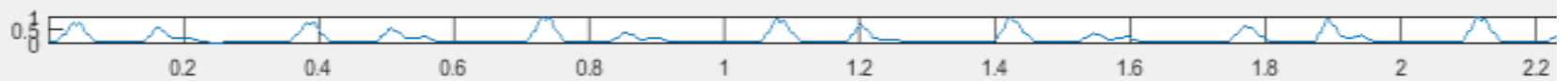

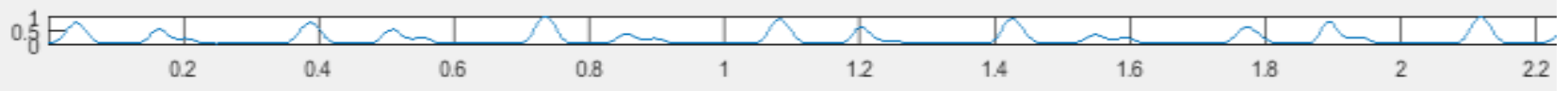
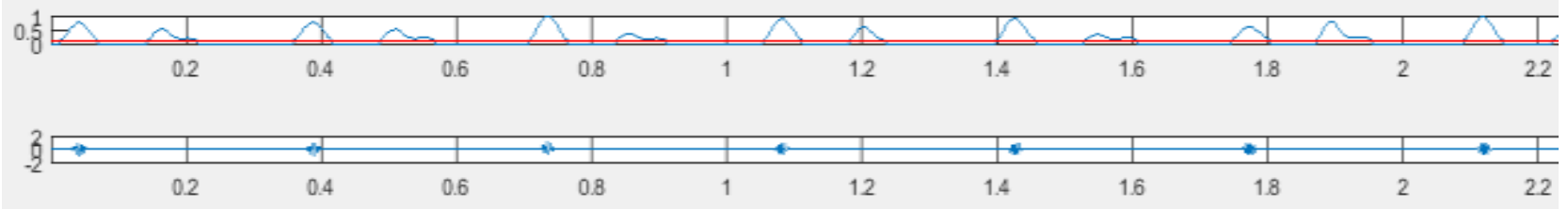

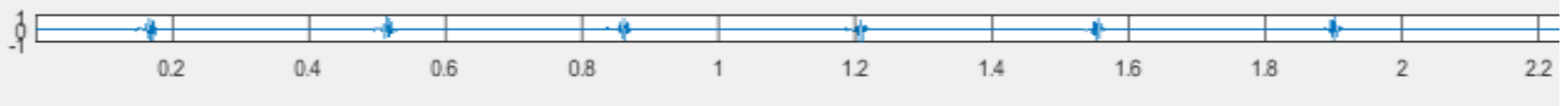

Fig.3.5 - (a)Denoise pcg signal (b) Time domain envelope (c) Frequency domain envelope(d) multiply time and frequency domain (e)apply GSF (f) apply thresolding (g)detected s1(h)detected s2 
Jamuna Kaushik et. al., Segmentation of Phonocardiograms Signal

3.6 Low amplitude entropy based detected s1 and s2 heart sound

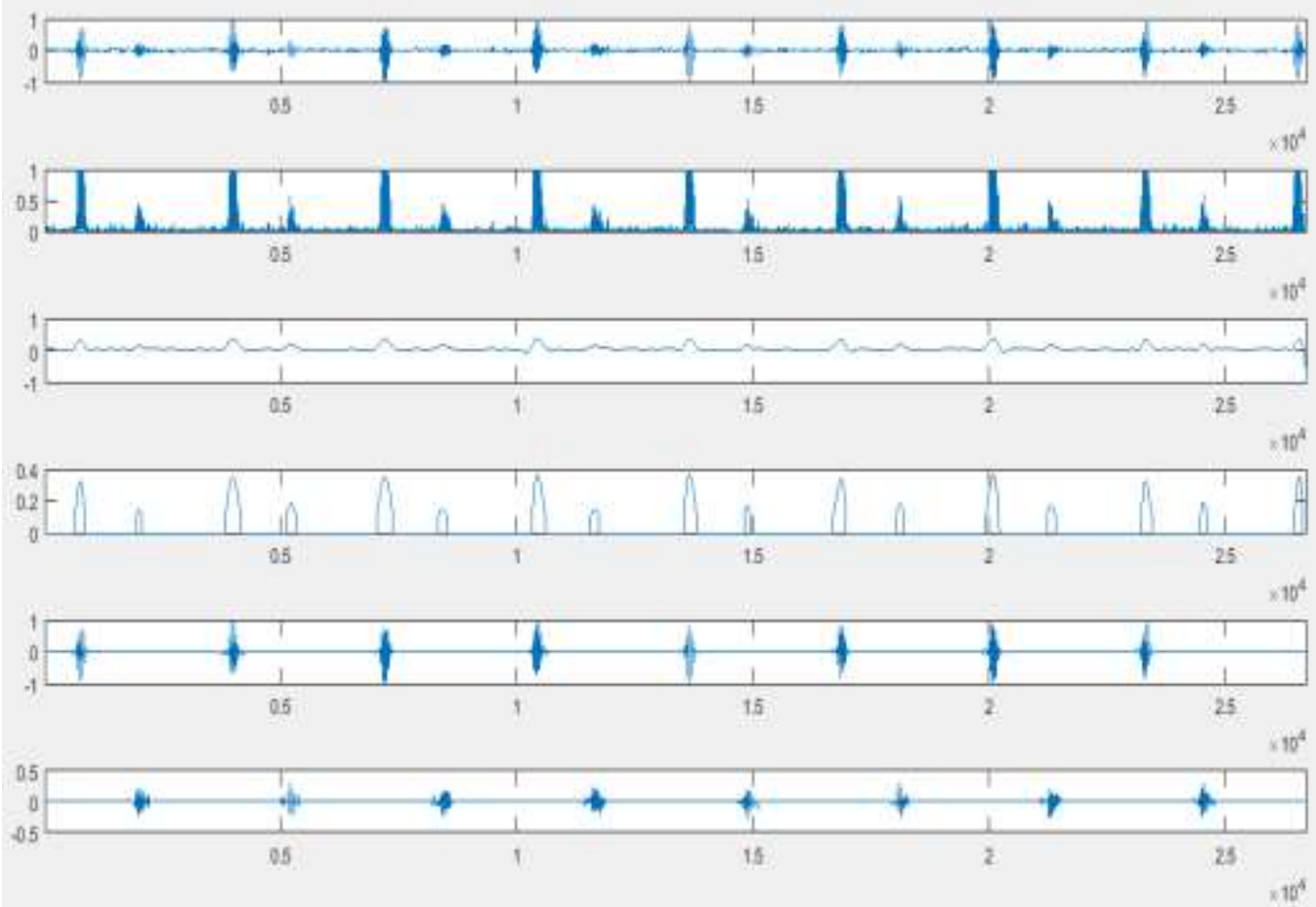

Fig.3.6- (a)Denoise pcg signal (b)low amplitude envelope (c) envelope detection (e)detected s1(f)detected s2

\subsection{Applying threshold level}

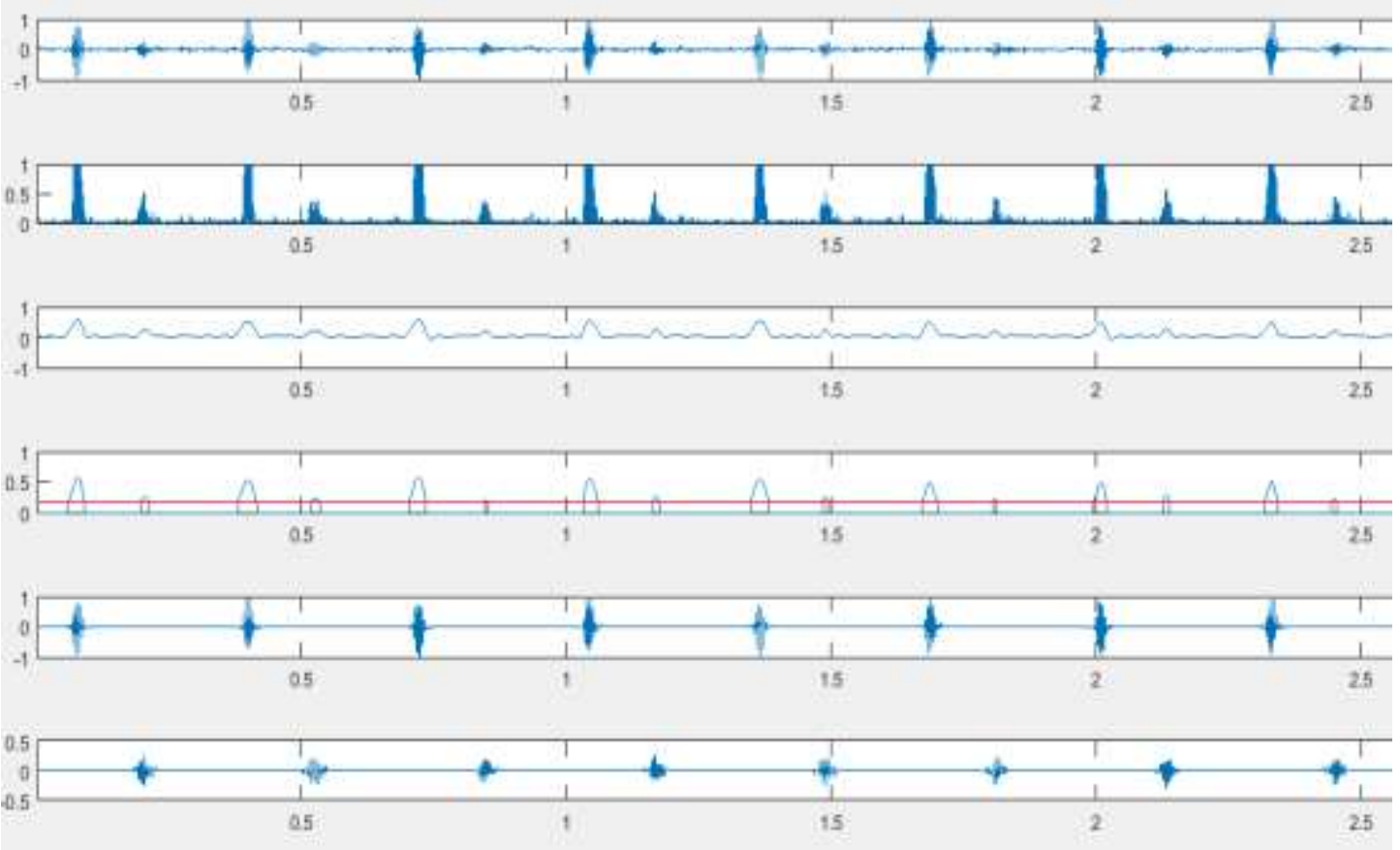

Fig.3.7- (a)Denoise pcg signal (b)low amplitude envelope (c) envelope detection(d) threshold decide (e)detected s1(f)detected s2

\section{PROBLEM IDENTIFICATION}

The immense information provided by the literature surveyed provided knowledge of problems that can affect the proposed work are as follows.

1.The PCG signal that has been taken as input may be of different sizes which may affect the accurate segmentation of signal. 
2.The sampling frequency of the signal may vary at the time of signal acquisition this may also affect.

3.The method of segmentation has to be chosen very carefully, as if not chosen properly may not segment the signal properly.

\section{CONCLUSION}

1. Segmentation accuracy is $100 \%$ which is better than almost all method used before.

2. Final classification accuracy, using 6 beats for heart signals, are $100 \%$.

3. Much better SNR.

\section{REFERENCES}

[1] Ali ,Denoising of Heart Sound Signals Using Discrete Wavelet Transform. Circuits, Systems, and Signal Processing, 44824497. IEEE,(November 2017).

[2] CHAKIR,Detection and Identification Algorithm of the S1 and S2 Heart Sounds, IEEE, (2016).

[3] NASERI,Detection and Boundary Identification of Phonocardiogram Sounds Using . an Expert Frequency-Energy Based Metric. Annals of Biomedical Engineering,279-292,IEEE(February 2013).

[4] Roy, rincipal Component Analysis (PCA) Approach to Segment Primary Components from Pathological Phonocardiogram . IEEE, 911-914,(2014).

[5] Saha, Classification of heart sounds using empirical mode decomposition based features . Int. J. Medical Engineering and Informatics, 1-18, (2008).

[6] Sun, An Improved Empirical Mode DecompositionWavelet Algorithm for Phonocardiogram Signal Denoising and its Application in the Frist and Second Heart Sound Extraction. IEEE , 187-191, (2013).

[7] Wai-Chi Fangl, An Efficient and Accurate Empirical Mode Decomposition of the Technical Design and Methods for Biological Sound . Industrial Technology Research Institute, Biomedical Technology and Device Research Laboratories, Medical Electronics \& Imaging Technology Division, Hsinchu, Taiwan (R.O.c.), 1-4.(2010).

[8] L Hamza Cherif, S M Debbal, F Bereksi-Reguig, Segmentation of heart sounds and heart mur-murs, J.Mechanics in Medicine and Biology, vol 8, Issue 4, P 549-559, (2008).

[9] MB Malarvili, I Kamarulazam, S Hussain, D Helmi, Heart Sound Segmentation Algorithm Based on Instantaneous Energy of Electrocardiogram, J. IEEEComputer in Cardiology, vol.30, p327-330, (2003).

[10] D Kumar, P Carvalho, M Antunes, J Henriques, M Maldonado, R Schmidt, J Ha- betha, Wavelet Transform And Simplicity Based Heart Murmur Segmentation, J. IEEE Computer in Cardiology, vol.33, p173-176,(2006).

[11] A.Gavrovska, M. Slavkovic, I. Reljin, B.Reljin, Application of wavelet and EMD based de-noising to phonocardiograms, J. IEEE,(2013).

[12] C.D.Papadaniil, L. J. Hadjleontiadis, Ecient heart sounds segmentation and ex- traction using ensemble empirical mode decomposition and kurtosis feature, J. of biomedical and health infor-matics, (2013).

[13] S.R.Messer, J. Agzarian, D. About, Optimal wavelet denoising for phonocardiograms, microe-lectronics journal, vol32 , 931-941.(2001).

[14] S.Mallat, A theory for multiresolution signal decomposition: the wavelet decomposition, IEEE Pattern Analysis and Machine Intelligence 11 (7) 867-870(1989).

[15] B.B Hubbard, The world According toWavelets, A K Peters Lts, 289 Linden Stree, Wellesley, MA 02181, (1996). 
Jamuna Kaushik et. al., Segmentation of Phonocardiograms Signal

[16] S.Mallat, Z.Zhang,Matching pursuits with time-frequency directories, IEEE Transaction on signal processing 41 3397-3415, (1993).

[17] M. Misiti, Y. Misiti, G. Oppenheim, J.-M. Poggi,Wavelet toolbox: For Use With Matlab, The Math Works Inc, (1996).

[18] A.Castro, T. V. Vinhoza, S. Mattos and M T. Coimbra, Heart sound segmentation of pediatric auscultations using wavelet analysis, IEEE EMBS, 3909-3912,(2013).

[19] F. Meziani, S.M Debbal, and A.Atbi, Analysis of phonocardiogram signals using wavelet trans-form, J. Med.Eng.Technol, Vol.36, no.6, pp.283-302 (2012)

[20] J.C. Chan, H.Ma, T.K.Saha, A novel level-based automatic wavelet selection scheme for partial discharge measurement, IEEE conference AUPEC, pp 1-6, (2012).

[21] Li, J., Jiang T., Grzybowski S., and Cheng C., Scale dependent wavelet selection for denoising of partial discharge detection, IEEE Transactions on Dielectrics and electrical insulation, vol.17, pp 1705-1714,(2010). 